Pacific Journal of Mathematics

ON FUNCTION FAMILIES WITH BOUNDARY 


\section{ON FUNCTION FAMILIES WITH BOUNDARY}

\section{JÓZEF SICIAK}

1. Introduction. Let $A$ be a family of real valued upper semicontinuous functions defined on a compact Hausdorff space $E$.

A closed set $F \subset E$ is called determining for $A$ if every function $f \in A$ attains its maximum on $F$. If for the space $E$ there exists one and only one minimal determining $F=F(E, A)$ (i.e., a determining set such that no proper closed subset of it is determining), then $F$ is called the boundary of $E$ with respect to the family $A$.

A function $h \in A$ is called a barrier-function of $A$ at a point $\stackrel{\circ}{x} \in F=$ $F(E, A)$ if and only if $h(\stackrel{\circ}{x})>h(x)$ for $x \neq \stackrel{\circ}{x}, x \in F$.

A point $\stackrel{x}{x} \in F$ for which there is a barrier-function of $A$ is called a semiregular boundary point of $E$ with respect to $A$. If for a point $\stackrel{\ominus}{x} \in F$ there exists a continuous (at the point $\stackrel{\circ}{x}$ ) barrier-function, then $\stackrel{\circ}{x}$ will be called a regular boundary point of $E$ with respect to $A$.

Let $D$ be a set contained in a topological space and let $f(x)$ be a real function defined on $D$. Then the function $f^{*}$ defined in the closure $\bar{D}$ of $D$ by means of

$$
f^{*}(x)=\lim _{x^{\prime} \rightarrow x} \sup f\left(x^{\prime}\right),
$$$$
x^{\prime} \in D, x \in \bar{D},
$$

is called an upper regularization of $f$.

Let $A_{1}$ be a subfamily of $A$. Then the function

$$
\varphi(x)=\left\{\sup _{f \in A_{1}} f(x)\right\}^{*},
$$

is called the upper envelope of $A_{1}$.

Let $f$ be an upper semicontinuous nonnegative function defined in a compact set $E$. We shall denote by $\|f\|_{E}$ the maximum of $f$ on $E$, $\|f\|_{E}=\max _{x \in E} f(x)$.

We say that a family $A$ of functions $f$ defined on $E$ is separating (or $A$ separates the points of $E$ ) if for any two points $x_{1} \neq x_{2}$ of $E$ there is a function $f \in A$ such that $f\left(x_{1}\right) \neq f\left(x_{2}\right)$.

A well known theorem of Silov [5] asserts: If $A$ is a family of absolute values of all functions of a separating algebra of complex continuous functions defined on a compact Hausdorff space $E$, then $E$ has the boundary $F$ with respect to the family $A$.

This boundary is sometimes called a Šilov boundary of $E$ (with respect to the given algebra).

E. Bishop [3] has recently proved that if $E$ is metrizable and $A$ is a complete (with respect to the uniform convergence) Banach algebra

Received May 30, 1961. This paper was prepared partially under the sponsorship of NSF Grant 10375. 
of continuous function on $E$, then the Šlov boundary of $E$ is the closure of regular points of $E$ with respect to $A$.

Let us mention that the papers of S. Bergman [1], [2] on the domains with a distinguished boundary surface are the first to indicate the significance of the boundary of a domain $D$ with respect to the algebra of holomorphic functions of several complex variables in $D$.

Recently it appeared that the notion of the boundary of a set with respect to the family of functions, which do not necessarily form an algebra, may be useful. For instance, Bremermann [4], considering a generalized solution of the Dirichlet boundary value problem within the family of pluri-subharmonic functions in a domain $D$ of the space $C^{n}$ of $n$ complex variables, had to consider the boundary of $D$ with respect to the family of pluri-subharmonic functions in $D$. The boundary values, in the procedure described by Bremermann, could be given just on the Silov boundary of $D$ and nowhere else. But the family of pluri-subharmonic functions does not form any algebra. Also in the case of the first boundary value problem for the heat conduction equation $u_{x x}-u_{t}=0$ in a domain $D$, the boundary values can be given only on a part of the boundary of $D$. That part is a Silov boundary of $D$ with respect to the solutions of the inequality $u_{x x}-u_{t} \geqq 0$. Those solutions do not form any algebra, of course.

The aim of this paper is to prove the existence of the boundary with respect to function families much more general than the algebras, namely, for the families $A$ which are closed only under the multilplication or addition of functions of $A$.

This fact can be applied to a uniform treatment of a Perron procedure of upper envelopes with respect to various function families having the boundary. Suppose that for a function family $A$ there exists a boundary $F=F(E, A)$. Then, by means of reasoning classical in potential theory, we have the following theorem:

If, along with $f$ and $g$, the family $A$ contains $\alpha f+\beta g$, where $\alpha \geqq 0$ and $\beta \geqq 0$, if $A$ contains all real constants, and if $\stackrel{\circ}{x} \in F$ is a regular boundary point of $E$ with respect to $A$ then for any real function $b(x)$ defined and continuous on $F$ we have

$$
b(\stackrel{\circ}{x})=\lim _{x \rightarrow \infty} \varphi(x),
$$

where $\varphi(x)$ denotes the upper envelope of all functions $f \in A$ such that $f(x) \leqq b(x)$ for $x \in F$.

Let any point of $F$ be regular. Then $\varphi(x)=b(x)$ for $x \in F$, and it is quite natural to look at such an upper envelope $\mathcal{P}(x)$ as at a generalized solution of the Dirichlet boundary value problem within the family $A$. If the function faraily $A$ is closed under ths operation of taking the 
upper envelope, then the generalized solution is a function of $A$.

There are well known examples of function families (which are not any algebra) within which the solution of the Dirichlet problem was found just by means of the Perron procedure [4], [6].

2. Some general function families with boundary. We shall need the following general

Lemma 1 (Silov). For any family $A$ of upper semicontinuous functions defined on a compact Hausdorff space $E$ there exists a minimal determining set (one at least).

This lemma can be proved by means of transfinite induction (see the proof by Silov [5]).

TheOREM 1. Let $A$ be a family of nonnegative functions defined and upper semicontinuous on a compact Hausdorff space $E$. If the family A satisfies the following conditions:

$1^{\circ}$ If $f$ and $g$ are functions of $A$ then the product $f \cdot g \in A$;

$2^{\circ}$ If $\hat{x}$ is an arbitrary fixed point of $E$ then for any neighborhood $U(\stackrel{\circ}{x})$ of $\stackrel{\circ}{x}$ and for any $\varepsilon>0$ there exists a finite system of functions $f_{1}, f_{2} \cdots, f_{k} \in A$ such that the set

$$
U^{*}=\left\{x \in E \mid f_{\mu}(x)<\varepsilon, \quad \mu=1,2, \cdots, k\right\}^{1}
$$

is contained in $U(\stackrel{\circ}{x})$ and $U^{*}$ contains a neighborhood $U^{\prime}(x)$ of $x$; then the set $E$ has a boundary with respect to $A .^{2}$

Proof. Due to Lemma 1 it is sufficient to prove that $E$ has only one minimal determining set with respect to $A$. The proof of the uniqueness may be given by a literal repetition of Silov's proof in [5]. This repetition is possible because Silov used only the assumptions formulated in Theorem 1.

REMARK. If $c$ is a positive real number and $f(x)$ is any real function upper semicontinuous on a closed set $E$, then the functions $\mathrm{c} \cdot \mathrm{f}$ and $f$ attain their maxima at the same points of $E$. Therefore, $E$ has a boundary with respect to $A$ if and only if $E$ has a boundary with respect to $\widetilde{A}$, where $\widetilde{A}$ denotes the family of functions $g$ which can be written in the form $g=c \cdot f, c>0, f \in A$.

The function family $A$ considered in Theorem 1 is closed under the operation of multiplication of functions of $A$. A similar theorem holds

1 The integer $k$ mas depend on $\dot{x}, t(x)$ or $\hat{a}$.

2 The similar theorem has been proved in [ 7$]$. 
for function families closed with respect to additions of functions of $A$.

THEOREM 1'. Let $A$ be a family of upper semicontinuous functions defined on a compact Hausdorff space E. If A satisfies conditions:

$1^{\circ}$ If $f, g \in A$, then $f+g \in A$;

$2^{\circ}$ If $\hat{x} \in E$ and $U(\stackrel{\circ}{x})$ is a neighborhood of $\hat{x}$ and $\varepsilon>0$, then there exists a finite system of functions $f_{1}, f_{2}, \cdots, f_{k} \in A$ such that

$$
U^{*}=\left\{x \mid e^{\rho_{\mu}(x)}<\varepsilon, \mu=1, \cdots, k\right\}
$$

is contained in $U(\stackrel{\circ}{x})$ and $U^{*}$ contains a neighborhood $U^{\prime}(\stackrel{\circ}{x})$ of $\stackrel{\circ}{x}$; then $E$ has a boundary with respect to $A$.

Proof. It is sufficient to observe that the family $A_{1}$ of functions $h=e^{f}$, where $f \in A$, satisfies the assumptions of Theorem 1 . Therefore, $E$ has a boundary with respect to $A_{1}$. But this is also a boundary of $E$ with respect to $A$.

REMARK 1. Any function family, which satisfies $2^{\circ}$, separates the points of $E$, but the converse statement is not true. For instance, let $E$ be the segment $[0,1]$ and let $A$ be the family of all powers $x^{\mu}, \mu=$ $1,2, \cdots$. Then $A$ satisfies $1^{\circ}$ but it does not satisfy $2^{\circ}$, although $A$ separates the points of $E$. The boundary of $E$ with respect to $A$ is in this case the only point $x=1$.

Now we shall prove the existence of the boundary for function families which are closed with respect to multiplication (or addition) and which only, instead of $2^{\circ}$ in Theorem 1 , separate the points of $E$. But we now must assume that the space $E$ is metric and the functions considered are continuous, while in Theorem 1 they could be only upper semicontinuous.

THEOREM 2. Let A be a family of nonnegative continuous functions defined on a compact metric space $E$. If $A$ satisfies the conditions:

$1^{\circ}$ If $f, g \in A$, then $f \cdot g \in A$;

$2^{\circ} \quad A$ separates the points of $E$;

then $E$ has a boundary with respect to $A .^{3}$

Proof. In virtue of Lemma 1 it is sufficient to prove only the uniqueness of the minimal determining set for $A$. For the proof per reductio ad absurdum let us assume that there exist two different minimal determining sets $F_{1}$ and $F_{2}$ for $A$. The set $F_{1} \mid F_{2}$ is nonempty, since, otherwise, we would have $F_{1} \subset F_{2}$ and $F_{2}$ would not be a minimal determining set. Let $\stackrel{\circ}{x}_{1} \in F_{1} \mid F_{2}$ and let $U_{1}\left(\stackrel{\circ}{x}_{1}\right)=\left\{x \in E \mid \rho\left(x, \stackrel{\circ}{x_{1}}\right)<1 / 2^{n_{1}}\right\}$ be

${ }^{3}$ After submitting this paper for publication, the author discovered that $\mathrm{H}$. Bauer, with different techniques, obtained more general results, see H. Bauer, Silovscher Rand und Dirichletsches Problem, Ann. L'Inst. Fourier XI (1961), 89-136. 
a neighborhood of $\stackrel{\circ}{x}_{1}$, where $n_{1}$ is an integer so large that $U_{1}\left(\stackrel{\circ}{x}_{1}\right) \cap F_{2}=\phi$. Since $F_{1}$ is a minimal determining set for $A$, there exists a function $f \in A$ which attains its maximum $m=\|f\|_{F_{1}}$ on $F_{1}$ in the set $U_{1}$, and such that $\max _{x \in U_{1}} f(x)=m>f(y), y \in F_{1} \mid U_{1}$. In virtue of $1^{\circ}$ and the Remark on p. 377, we can assume that

$$
\|f\|_{F_{1}}=\|f\|_{F_{2}}=\|f\|_{t}=1, \quad f(y)<\frac{1}{4} \text { for } y \in F_{1} \mid U_{1} .
$$

Since $F_{2}$ is determining and $U_{1} \cap \mathrm{F}_{2}=\phi$, there must be a point $\stackrel{\vartheta}{y}_{1} \in F_{2}$ such that $f\left(\hat{y}_{1}\right)=1$. The function $f(x)$ is continuous. Thus, there is a neighborhood $V_{1}\left(\stackrel{\circ}{y}_{1}\right)=\left\{y \mid \rho\left(y, \hat{y}_{1}\right)<1 / 2^{m_{1}}\right\}$ of $\stackrel{\circ}{y}_{1}$, where $m_{1}$ is so large that

$$
f(x)>\frac{3}{4} \text { for } x \in V_{1} \text { and } \overline{V_{1}\left(\stackrel{\circ}{y}_{1}\right)} \cap \overline{U_{1}\left(\stackrel{O}{x}_{1}\right)}=\phi .
$$

Since $F_{2}$ is minimal there is a function $g(x)$ such that $\|g\|=g\left(y_{1}\right)=1$, $y_{1}$ being a point of $V_{1}$, and $g(x)<1 / 4$ for $x \in F_{2} \mid V_{1}$. Now we put $h(x)=$ $f(x) g(x)$. We can easily verify that

$$
h\left(y_{1}\right) \geqq \frac{3}{4}, h(x)<\frac{1}{4} \text { for } x \in F_{1} \mid U_{1} \quad \text { or } \quad x \in F_{2} \mid V_{1} .
$$

Since $h\left(y_{1}\right) \geqq 3 / 4$, so $\max _{x \in J_{1}} h(x)=\|h\|_{E} \geqq 3 / 4$. Therefore, the function $h_{1}(x)=\left[h(x) /\|h\|_{E}\right]^{k}$, where $k$ is a sufficiently large integer, satisfies the conditions,

$$
\left\|h_{1}\right\|_{E}=1 ; h_{1}(x)<\frac{1}{4} \text { for } x \in F_{1} \mid U_{1} \quad \text { or } \quad x \in F_{2} \mid V_{1},
$$

and moreover there exists a point $x_{1} \in U_{1}$ and a point $y_{1} \in V_{1}$ for which $h_{1}\left(x_{1}\right)=h\left(y_{1}\right)=1$.

This was the first step of our proof. To begin the next one, let us observe that one can find an integer $n_{2}>n_{1}$ so large that

$$
U_{2}\left(x_{1}\right)=\left\{x \mid \rho\left(x, x_{1}\right)<\frac{1}{2^{n_{2}}}\right\} \subset U_{1}\left(\stackrel{\circ}{1}_{1}\right) \text { and } h_{1}(x) \geqq \frac{3}{4} \quad \text { for } x \in U_{2} .
$$

Since $F_{1}$ is a minimal determining set there exists $f_{0} \in A$ such that $\left\|f_{0}\right\|_{E}=1, f_{0}(x)<1 / 4$ for $x \in F_{1} \mid U_{2}$ and $f_{0}\left(\hat{x}_{2}\right)=1$ for a point $\stackrel{\circ}{x} \in U_{2}$. We define $f_{1}(x)=f_{0}(x) h_{1}(x)$. We have $f_{1}(x)<1 / 4$ for $x \in F_{1} \mid U_{2}$ or $x \in F_{2} \mid V_{2}$ and $f_{1}\left(\stackrel{\circ}{2}_{2}\right) \geqq 3 / 4$. So $\left\|f_{1}\right\|_{E} \geqq 3 / 4$ and there is a point $y_{2} \in V_{1}$ such that $f_{1}\left(\stackrel{\circ}{2}_{2}\right)=\left\|f_{1}\right\|_{E}$. Therefore, the function $f=\left(f_{1} /\left\|f_{1}\right\|_{E}\right)^{k}, k$ being a suitable integer, satisfies the conditions

$$
|f| \mid=f\left(y_{2}\right)=1 \quad \text { and } \quad f(x)<\frac{1}{4} \quad \text { for } x \in F_{1} \mid U_{2} \quad \text { or } \quad x \in F_{2} \mid V_{1} .
$$

The function $f(x)$ is continuous, so one can find an integer $m_{2}>m_{1}$ so large that $V_{2}\left(y_{2}\right)=\left\{y \mid \rho\left(y_{1}, y_{2}\right)<1 / 2^{m_{2}}\right\} \subset V_{1}$ and $f(y) \geqq 3 / 4$ for $y \in V_{2}$. 
Since $F_{2}$ is minimal, there is a function $g \in A$ and a point $\stackrel{\circ}{2}_{2} \in V_{2}\left(y_{2}\right)$ such that $\|g\|_{E}=g\left(\breve{y}_{2}\right)=1$ and $g(y)<1 / 4$ for $F_{2} \mid V_{2}$. Therefore, the function $h(x)=f(x) g(x)$ has the following properties: $h \in A,\|h\| \geqq 3 / 4, h(x)<1 / 4$ for $x \in F_{1} \mid U_{2}$ or $x \in F_{2} \mid V_{2}$. Hence, the function $h_{2}(x)=\left(h(x) /\|h\|_{E}\right)^{k}$, where $k$ is a snfficiently large integer, satisfies

$$
\left\|h_{2}\right\|_{E}=1, \quad h_{2}(x)<\frac{1}{4} \text { for } x \in F_{1} \mid U_{3} \text { or } x \in F_{2} \mid V_{2},
$$

and there is a point $x_{2} \in U_{2}$ and a point $y_{2} \in V_{2}$ for which $h_{2}\left(x_{2}\right)=h_{2}\left(y_{2}\right)=1$.

Continuing this procedure, we construct two sequences of points $\left\{x_{\nu}\right\}$ and $\left\{y_{\nu}\right\}$, two descending sequences of neighborhoods $\left\{U_{\nu}\left(x_{\nu}\right)\right\}$ and $\left\{V_{\nu}\left(y_{\nu}\right)\right\}$, and a sequence of functions $\left\{h_{\nu}(x)\right\}$. By their construction these sequences satisfy the following conditions: The functions $h_{\nu}(x), \nu=1,2, \cdots$ belong to $A$ (in fact, we have only $h_{\nu}(x)=c_{\nu} h_{\nu}^{*}(x)$, where $h_{\nu}^{*} \in A$ and $c_{\nu}=$ const $>0$, but it does not matter because of the Remark on p. 377). The neighborhoods $U_{\nu}$ and $V_{\nu}$ converge to points $\stackrel{\circ}{x}$ and $\stackrel{y}{y}$, respectively. The points $\dot{x}$ and $\dot{y}$ are also the limits of $\left\{x_{\nu}\right\}$ and $\left\{y_{\nu}\right\}$, respectively. For any $\nu=1,2, \cdots$ we have

$$
\left\|h_{\nu}\right\|_{E}=h_{\nu}\left(x_{\nu}\right)=h_{\nu}\left(y_{\nu}\right)=1, \quad h_{\nu}(x)<\frac{1}{4} \text { for } x \in F_{1} \mid U_{\nu} \text { or } x \in F_{2} \mid V_{\nu} .
$$

Since $U_{\nu+1} \subset U_{\nu}, V_{\nu+1} \subset V_{\nu}$ and $U_{\nu} \cap V_{\nu}=\phi, \nu=1,2, \cdots$, we have

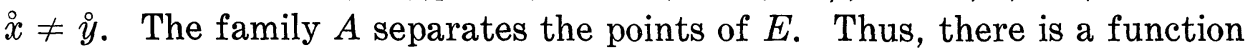
$h \in A$ such that $h(\stackrel{\circ}{x} \neq h(\stackrel{\circ}{)}$. Without any loss of generality we may assume that $h(\stackrel{\circ}{x})<h(\grave{y})$. Let $h(\stackrel{\circ}{y})-h(\stackrel{\circ}{x})=3 \varepsilon$. Since $h(x)$ is continuous, we may find two neighborhoods $U(\stackrel{\circ}{x})$ and $V(\stackrel{\circ}{y})$ such that

$$
h(x)<h(\stackrel{x}{x})+\varepsilon \text { for } x \in U(\stackrel{x}{)} \text { and } h(\grave{y})-\varepsilon<h(y) \text { for } y \in V(\grave{y}) \text {. }
$$

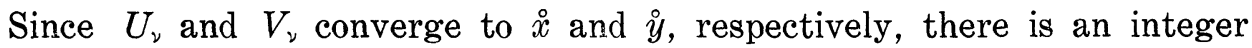
$\nu_{0}$ such that $U_{\nu_{0}} \subset U(\stackrel{\circ}{x})$ and $V_{\nu_{0}} \subset V(\grave{y})$. Let $M=\|h\|_{E}$, and let $m$ be so large that $M / 2^{m}<\varepsilon$. Then the function $b(x)=h(x)\left[h_{\nu_{0}}(x)\right]^{m}$ satisfies the conditions:
(i) $b(x) \leqq \frac{M}{4^{m}}<\varepsilon$, for $x \in F_{1} \mid U_{\nu_{0}}$ or $x \in F_{2} \mid V_{\nu_{0}}$;
(ii) $b(x)<h(\stackrel{\circ}{x})+\varepsilon$, for $x \in U_{\nu_{0}}$;
(iii) $b(x) \geqq h(\stackrel{\circ}{y})-\varepsilon h(\stackrel{\circ}{x})+\varepsilon$, for $x \in V_{\nu_{0}}$.

Thus the function $b(x)$ attains its maximum $\|b\|_{E}$ on $F_{2}$ and $b(x)<\|b\|_{E}$ for $x \in F_{1}$. Therefore, $F_{1}$ is not a determining set. This contradiction completes our proof.

A simple consequence of Theorem 2 is the following 
THEOREM 2'. Let $A$ be a separating family of real continuous functions defined on a compact metric space $E$, and let $A$ be closed under the addition operation. Then $E$ has a boundary with respect to $A$.

3. Regular boundary points. The following theorem is a reformulation of the theorem by E. Bishop (see [3], p. 633) in a slightly more general form.

THEOREM 3. If $A$ is a separating family of nonnegative continuous functions defined on a compact metric Hausdorff space $E$ and if

$1^{\circ}$ A contains positive constants,

$2^{\circ} \quad A$ is closed under addition and multiplication of functions of $A$,

$3^{\circ}$ A contains limits of uniformly convergent sequences of functions of $A$;

then $E$ has a boundary $F$ with respect to $A$, and $F$ is the closure of regular boundary points of $E$ with respect to $A$.

Proof. The boundary $F$ exists by Theorem 2. Let $x_{0}$ be a fixed point of $F$ and let $U_{v}=U(\stackrel{\circ}{)})$ be a neighborhood of $U_{v}$. It will now be our task to find a regular point in the neighborhood $U_{0}$. By the definition of $F^{\prime}$ and in virtue of assumptions $1^{\circ}$ and $2^{\circ}$ of the theorem, there is a function $f_{0} \in A$ such that $\left\|f_{0}\right\|_{E}=1, f_{0}\left(x_{1}\right)=1$ for some point $x_{1} \in U_{0}$, and $f_{0}(y)<1 / 4$ for $y \in F \mid U_{0}$. Let $U_{1} \subset U_{0}$ be a neighborhood of $x_{1}$ such that $f_{0}(x)>3 / 4$ for $x \in U_{1}$. There is a function $f_{1}(x) \in A$ such that $\left\|f_{1}\right\|_{E}=1, f_{1}\left(x_{2}\right)=1$ for a point $x_{2} \in U_{1}$, and $f_{1}(y)<1 / 4$ for $x \in F \mid U_{1}$. Repeating this procedure, we can define:

$1^{\circ}$ a sequence of neighborhoods $\left\{U_{\nu}\right\}, U_{\nu+1} \subset U_{\nu}$, whose product contains a single point $y_{0}$,

$2^{\circ}$ a sequence of functions $f_{\nu}(x)$ such that

$f_{\nu}\left(y_{0}\right)>\frac{3}{4}, f_{\nu}(y)<\frac{1}{4} \quad$ for $y \in F \mid U_{\nu} \quad$ and $\quad\left\|f_{\nu}\right\|_{E}=1, \nu=1,2, \cdots$.

Now we can define a function $g \in A$, which is a barrier function of $A$ at the point $y_{0}$. Namely, in the same way as in the proof of Theorem 2 of [3], we at first construct, by induction, a sequence of functions $g_{n} \in A$ such that

(i) $\left\|g_{n+1}-g_{n}\right\|_{E}<2^{-n+1}$
(ii) $\left\|g_{n}\right\|_{E} \leqq 3\left(1-2^{-n-1}\right)$
(iii) $g_{n}\left(y_{0}\right)=3\left(1-2^{-n}\right)$
(iv) $\left|g_{n+1}(y)-g_{n}(y)\right|<2^{-n-1}$ for $y \in F \backslash U_{\mu_{n+1}}$, 
where $\left\{U_{\mu_{n+1}}\right\}$ is a suitably chosen subsequence of $\left\{U_{n}\right\}$.

We put $g_{1}(x)=3 / 2\left[f_{1}\left(y_{0}\right)\right]^{-1} f_{1}(x)$ and check that $g_{1}$ satisfies (ii) and (iii). Assuming that $g_{1}, \cdots, g_{k}$ have been constructed, we define $g_{k+1}$ in the following way. Since $g_{k}$ is continuous and $g_{k}\left(y_{0}\right)=3\left(1-2^{-k}\right)$, we can find $\mu_{k+1}>\mu_{k}$ such that

$$
g_{k}(x)<3\left(1-2^{-k}\right)+2^{-k-2} \text { for } x \in U_{\mu_{k+1}} .
$$

Now we define $g_{k+1}(x)=g_{k}(x)+3 \cdot 2^{-k-1}\left[f_{\mu_{k+1}}\left(y_{0}\right)\right]^{-1} f_{\mu_{k+1}}(x)$ and check that $g_{1}, g_{2}, \cdots, g_{k}, g_{k+1}$ satisfy (i)-(iv) (for details see [3], p. 633). A barrier function of $A$ at the point $\hat{y}$ is given by

$$
g(x)=3 \sum_{k=1}^{\infty} \frac{f_{\mu_{k}}(x)}{2^{k} f_{\mu_{k}}\left(y_{0}\right)}=\lim _{n \rightarrow \infty} g_{n}(x), \quad x \in E .
$$

Namely, we have $g\left(y_{0}\right)=3,\|g\|_{E} \leqq 3$. Since

$$
g(x)=g_{n}(x)+3 \sum_{k=n+1}^{\infty} \frac{f_{\mu_{k}}(x)}{2^{k} f_{\mu_{k}}\left(y_{0}\right)}
$$

and since $f_{\mu_{k}}(x)<1 / 4$ for $x \in F \mid U_{\mu_{k+1}}$, we have

$$
g(x)<3\left(1-2^{-n-1}\right)+3 \sum_{k=n+1}^{\infty} \frac{1 / 4}{2^{k} \cdot 3 / 4}=3-2^{-n-1}<3 \text { for } x \in F \mid U_{\mu_{n+1}} .
$$

Hence, $g(x)<3$ for $x \neq y_{0}$.

4. Applications. Let $D$ be a bounded set in the Euclidean space $R^{n}, n \geqq 1$. Let $A=A(D)$ be a family of real functions defined on $D$. We denote by $A^{*}$ the set of all the upper regularizations of functions of $A$. We shall call the boundary of $\bar{D}$ with respect to $A^{*}$ also the boundary of $D$ with respect to $A$.

By means of Theorems 1 and 2, we can easily check that the following function families have boundaries:

(1) The family of moduli of all polynomials of $n$ complex variables for any bounded set $D \subset C^{n}$.

(2) The family of moduli of holomorphic functions of $n$ complex variables for any bounded set $D \subset C^{n}$.

(3) The family of pluri-subharmonic functions for any bounded set $D \subset C^{n}$.

(4) The family of convex functions of $n$ real variables for any bounded domain $D \subset R^{n}, n \geqq 1$.

(5) The family of solutions in a bounded domain $D \subset R^{n}$ (continuous in $\bar{D}$ ) of the system of differential inequalities (or equalities)

$$
\mathscr{E}^{(i)}[u]=\sum a_{\mu_{1}}^{(i)}, \ldots, \mu_{n} \frac{\partial^{\mu} u}{\partial x_{1}^{\mu_{1}} \cdots \partial x_{n}^{u} n} \geqq 0, \begin{aligned}
& k \geqq \mu_{1}+\cdots+\mu_{n}=\mu \geqq 2 \\
& i=1,2, \cdots, l
\end{aligned}
$$


(6) The family of continuous solutions of the system of differential inequalities

$$
\begin{gathered}
P[u]=\sum a_{\mu_{1}}^{(i)}, \ldots, \mu_{n} \frac{\partial^{\mu} u}{\partial x_{1}^{\mu_{1}} \cdots \partial x_{n}^{\mu_{n}}}+\sum_{\nu=1}^{m} b_{\nu} \frac{\partial u}{\partial y_{\nu}} \geqq 0, \\
k \geqq \mu \geqq 2, \quad b_{\nu}=\text { const } \quad i=1,2, \cdots, l
\end{gathered}
$$

in any bounded domain $D \subset R^{n+m}$ contained in the set

$$
S=\left\{\left.\left(x_{1}, \cdots, x_{n}, y_{1}, \cdots, y_{m}\right)\right|_{i=1,2, \cdots, n} ^{-\infty<x_{\imath}<\infty}, \begin{array}{c}
y_{j} \in\left[0, \varepsilon_{j} \cdot \infty\right) \\
j=1,2, \cdots, m
\end{array}\right\},
$$

The statements of (1) and (2) follow from Theorem 1 with $f_{\mu}(z)=$ $a\left|z_{\mu}-z_{k^{0}}^{0}\right|, \mu=1,2, \cdots, n$ where $a=$ const is suitably chosen. (3) follows from Theorem $1^{\prime}$ with $f_{\mu}(z)=\log \left|a\left(z_{\mu}-z_{\mu}^{0}\right)\right|, \mu=1, \cdots, n$.

The families (4)-(6) are closed with respect to addition of their functions. The function $f(x)=\sum_{k=1}^{n}\left(x_{k}-x_{k}^{0}\right)^{2}$ is a universal separating convex function. The functions $f_{\mu}(x)=x_{\mu}-\grave{x}_{\mu}, \mu=1,2, \cdots, n$ are separating for (4) and (5). The functions $f_{\mu}(x)=x_{\mu}-\grave{x}_{\mu}, \mu=1,2, \cdots, n$, $g_{\mu}(y)=\varepsilon_{\mu}\left(y_{\mu}-\check{y}_{\mu}\right), \mu=1,2, \cdots, m$ are separating for (6).

Let us observe that the family (5) involves as a special case the family of double-harmonic functions. It is well known [1] that the boundary of a bicylinder with respect to double-harmonic functions is equal to the boundary of the bicylinder with respect to holomorphic functions. A similar situation holds for strictly pseudo-convex domains. But it is not known what is the situation for general domains. The relation between the Šlov boundary of a domain $D \subset C^{n}$ with respect to holomorphic functions and with respect to pluri-subharmonic functions has been investigated in [4].

The family (6) involves as a special case the family of "subparabolic" functions (compare [6]).

Any linear function $f(x)=a_{1} x_{1}+a_{2} x_{2}+\cdots a_{n} x_{n}+b$, where $a_{k}$ are real numbers, satisfies the system of inequalities $\mathscr{E}^{(i)}[f] \geqq 0, i=$ $1,2, \cdots, l$. Let $D$ be a strictly convex domain in the space $R^{n}$. This means that for any point $\grave{x} \in D^{\bullet}, D^{\cdot}$ being a topological boundary of $D$, there is a hyperplane $a_{1} x_{1}+\cdots+a_{n} x_{n}+b=0$ which has no common

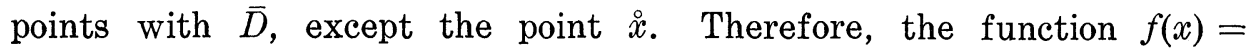
$a_{1} x_{1}+\cdots+a_{n} x_{n}+b$ (multiplied by -1 , if necessary) is a continuous barrier-function of family (5) at the point $\dot{x}$. Hence, by the theorem on p. 376 , we have

CoRollary. If $D$ is a strictly convex domain, then for any continuous function $b(x)$ defined on $D^{\cdot}$ there is a generalized solution $\varphi(x)$ of the Dirichlet boundary value problem inside any family (5). 
Particularly, if $D$ is strictly convex and $b(x)$ is continuous on $D \cdot$, then there is a convex function $\varphi(x)$, continuous in $\bar{D}$, such that $\varphi(x)=b(x)$ for $x \in D$.

THEOREM. If $D$ is a bounded domain in $C^{n}$, then the boundary of $D$ with respect to the family $A$ of pluri-subharmonic functions in $D$, continuous in $\bar{D}$, is the closure of regular points of $D$ with respect to $A$.

Proof. Let $A_{1}$ denote the family of all functions $g$ such that there exists a function $f \in A$ for which $g(x)=e^{f(x)}$. We can easily check that $A_{1} \subset A$ and $A_{1}$ satisfies all the assumptions of Theorem 3. Indeed, if $f$ and $g \in A_{1}$, then by a computation of the Hermitian form

$$
\sum_{\mu, \nu=1}^{n} \frac{\partial^{2} \log (f+g)}{\partial z_{\mu} \partial \bar{z}_{\nu}} \lambda_{\mu} \bar{\lambda}_{\nu}
$$

we check that $f+g \in A_{1}$ in the case of $f$ and $g$ being sufficiently regular. The general case is attained by approximation. The other assumptions follow directly from the known properties of pluri-subharmonic functions. By Theorem 1 domain $D$ has a boundary with respect to $A_{1}$. By Theorem 3 this boundary is the closure of the regular boundary points with respect to $A_{1}$. Let us now observe that the boundary $F$ of $D$ with respect to $A$ is the same as the boundary $F_{1}$ of $D$ with respect to $A_{1}$. Namely, since $A_{1} \subset A$, then $F_{1} \subset F$. The function $g$ and $e^{g}$ attain their maxima in the same points of $\bar{D}$. If $g \in A$, then $e^{g} \in A_{1}$. Thus any function of $A$ assumes its maximum on $F_{1}$, whence $F \subset F_{1}$. It follows that $F=F_{1}$, and the boundary of $D$ with respect to $A$ is the closure of regular boundary points of $D$ with respect to $A$.

\section{BIBLIOGRAPHY}

1. S. Bergman, Über die ausgezeichneten Randfä̈chen in der Theorie der Funktionen von zwei komplexer Veränderlichen, Math Ann., 104 (1931), 611-636.

2. - Über eine in gewissen Bereichen mit Maximumfläche gültige Integraldarstel. lung der Funktionen zweier komplexen Variablen, Math. Z., 39 (1934), 76-94, 605-608.

3. E. Bishop, A minimal boundary for function algebras, Pacific J. Math., 9 (1959), 629-642.

4. H. J. Bremermann, On a generalized Dirichlet problem for pluri-subharmonic functions and pseudoconvex domains. Charactcrization Šilov boundaries, Trans. Amer. Math. Soc., 91 (1959), 246-276.

5. J. M. Gelfand, D. A. Raikov, and G. E. Šilov, Commutative normed rings, Uspeki Mat. Nauk (N. S.) 1, 2 (12) (1946), 48-146.

6. J. Petrowski, Zur ersten Randwertaufgabe der Wärmeleitungsgleichung, Compositio Mathematica 1 (1935), 383-419.

7. J. M. Singer and R. Arens, Function values as boundary integrals, Proc. Amer. Math. Soc., 5 (1954), 735-745. 


\section{PACIFIC JOURNAL OF MATHEMATICS}

\section{EDITORS}

Ralph S. Phillips

Stanford University

Stanford, California

M. G. Arsove

University of Washington

Seattle 5, Washington
A. L. Whiteman

University of Southern Californla

Los Angeles 7, California

Lowell J. Paige

University of California

Los Angeles 24, California

\section{ASSOCIATE EDITORS}

E. F. BECKENBACH

D. DERRY

H. L. ROYDEN

E. G. STRAUS

T. M. CHERRY

M. OHTSUKA

E. SPANIER

F. WOLF

\section{SUPPORTING INSTITUTIONS}

UNIVERSITY OF BRITISH COLUMBIA

STANFORD UNIVERSITY

CALIFORNIA INSTITUTE OF TECHNOLOGY

UNIVERSITY OF CALIFORNIA

MONTANA STATE UNIVERSITY

UNIVERSITY OF TOKYO

UNIVERSITY OF UTAH

UNIVERSITY OF NEVADA

NEW MEXICO STATE UNIVERSITY

OREGON STATE UNIVERSITY

UNIVERSITY OF OREGON

OSAKA UNIVERSITY

WASHINGTON STATE UNIVERSITY

UNIVERSITY OF WASHINGTON

UNIVERSITY OF SOUTHERN CALIFORNIA

AMERICAN MATHEMATICAL SOCIETY CALIFORNIA RESEARCH CORPORATION SPACE TECHNOLOGY LABORATORIES NAVAL ORDNANCE TEST STATION 


\section{Pacific Journal of Mathematics}

\section{Vol. 12, No. $1 \quad$ January, 1962}

Jonathan L. Alperin, Groups with finitely many automorphisms $\ldots \ldots \ldots \ldots \ldots \ldots \ldots \ldots$

Martin Arthur Arkowitz, The generalized Whitehead product ................ 7

John D. Baum, Instability and asymptoticity in toplogical dynamics . . . . . . . . . . 25

William Aaron Beyer, Hausdorff dimension of level sets of some Rademacher series .... $\quad 35$

Frank Herbert Brownell, III, A note on Cook's wave-matrix theorem . . . . . . . . . . . . . 47

Gulbank D. Chakerian, An inequality for closed space curves ................. 53

Inge Futtrup Christensen, Some further extensions of a theorem of Marcinkiewicz ....... 59

Charles Vernon Coffman, Linear differential equations on cones in Banach spaces . . . . . 69

Eckford Cohen, Arithmetical notes. III. Certain equally distributed sets of integers . . . . . 77

John Irving Derr and Angus E. Taylor, Operators of meromorphic type with multiple poles

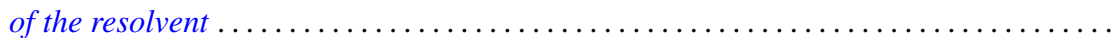

Jacob Feldman, On measurability of stochastic processes in products space .............

Robert S. Freeman, Closed extensions of the Laplace operator determined by a general class of boundary conditions, for unbounded regions ......................

Robert E. Fullerton, Geometric structure of absolute basis systems in a linear topological

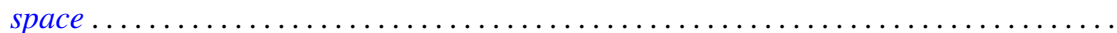

Dieter Gaier, On conformal mapping of nearly circular regions

Andrew Mattei Gleason and Hassler Whitney, The extension of linear functionals defined

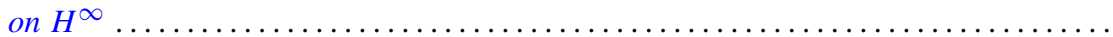

Seymour Goldberg, Closed linear operators and associated continuous linear

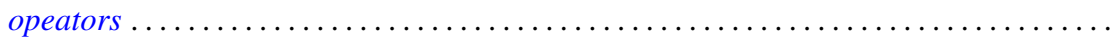

Basil Gordon, Aviezri Siegmund Fraenkel and Ernst Gabor Straus, On the determination of sets by the sets of sums of a certain order

Branko Grünbaum, The dimension of intersections of convex sets. .

Paul Daniel Hill, On the number of pure subgroups

Robert Peter Holten, Generalized Goursat problem . .

Alfred Horn, Eigenvalues of sums of Hermitian matrices ...........

Henry C. Howard, Oscillation and nonoscillation criteria for

$$
y^{\prime \prime}(x)+f(y(x)) p(x)=0
$$

Taqdir Husain, $S$-spaces and the open mapping theorem ...

Richard Eugene Isaac, Markov processes and unique stationary probability measures ...

John Rolfe Isbell, Supercomplete spaces ....................

John Rolfe Isbell, On finite-dimensional uniform spaces. II .........

N. Jacobson, A note on automorphisms of Lie algebras ..............

Antoni A. Kosinski, A theorem on families of acyclic sets and its applications

Marvin David Marcus and H. Minc, The invariance of symmetric functions of singular values...

Ralph David McWilliams, A note on weak sequential convergence.

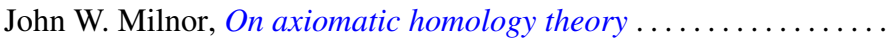

Victor Julius Mizel and Malempati Madhusudana Rao, Nonsymmetric projections in

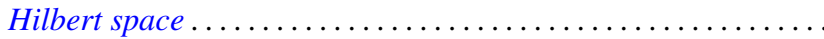

Calvin Cooper Moore, On the Frobenius reciprocity theorem for locally compact

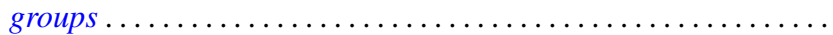

Donald J. Newman, The Gibbs phenomenon for Hausdorff means . 